\title{
Premature menopause secondary to Rathke's pouch cyst
}

\author{
Desai P, Ganeshanathan $\mathbf{S}^{1}$
}

\section{Summary}

A 35 year old female presented with 6 years of amenorrhoea with a background history of menstrual irregularity preceding the amenorrhoea. She was treated as polycystic ovarian syndrome (PCOS) but finally found to have a very large pituitary lesion. This was originally thought to be a non-functioning pituitary adenoma, however intraoperative findings suggest the lesion was likely a Rathke's cleft cyst, a relatively rare sellar mass whose hormonal effects were secondary to compression of normal pituitary tissue. Interestingly despite definitive resection and stabilisation of lactotrophs and gonadotroph levels our patient continued to have hypogondotrophic hypogonadism and finally required a hysterectomy for the safe initiation of HRT.

Background: Pituitary pathology accounts for $19 \%$ of secondary amenorrhoea. Because menstrual disturbances are a large part of the workload in gynaecology it is important for gynaecologists to have an understanding of pituitary aetiologies for amenorrhoea. As well as secondary amenorrhoea, pituitary pathologies can also mimic other gynaecological conditions such as polycystic ovarian syndrome and primary ovarian failure. It is important to consider the pituitary gland as a cause.

Gynaecologists are in a good position to recognise the presentation of many pituitary lesions. Approximately 1 in 3 pituitary adenomas are prolactinomas, making it the most common form of pituitary adenoma. High serum prolactin presents with subfertility, oligomenorrhoea or amenorrhoea, and interestingly, least commonly with galactorrhoea. Many of these symptoms would promote a referral to gynaecology. Other pituitary lesions can also present with these same symptoms purely by mass effect and compression of the pituitary stalk. Stalk compression causes decreased negative feedback by dopamine on prolactin production leading to hyperprolactinaemia. Therefore, the most common presentation of pituitary macroadenomas, both hormone secreting and non-functioning, are also menstrual and fertility dysfunction.

The mass effect caused by pituitary lesions is not limited to pituitary adenomas. Rarer lesions include craniopharyngiomas, meningiomas, malignant tumours, hypophsitis and Rathke's cleft cysts. The case I would like to put forward is of particular interest as our case study was found to have a large pituitary lesion which was thought to be a Rathke's cleft cyst, a relatively rare sellar mass, whose hormonal effects were all secondary to compression of normal pituitary tissue. Interestingly despite resection of the lesion our patient continued to have amenorrhea secondary to pituitary tissue damage. She illustrates the strong link between gynaecological symptoms and pituitary and sellar pathology, and is a rare and interesting learning case.

Department of Obstetrics and Gynaecology Logan Hospital, Metro South Health Network, Meadowbrook, Queensland.

Correspondence: Sabaratnam Ganeshanathan

E-mail: ganeshananthan@gmail.com

Competing interests: None ment for management of PCOS diagnosed by private gynaecologist when she presented with excessive facial hair, irregular periods and multiple ovarian cysts.

In September 2010 the patient presented to endocrinologists with optic chiasm compression and visual field defects as well as pituitary adrenal axis dysfunction, hypothyroidism, amenorrhoea and glactorrhoea. The patient was diagnosed with a pituitary tumour, presumed to be a non-functioning pituitary macroadenoma. MRI revealed a $31 \times 28 \mathrm{~mm}$ pituitary cystic pituitary macroadenoma expanding the sellar turcica and compressing the undersurface of the optic chiasm. A trans-phenoidal surgery was performed on 14/12/10. Interestingly intraoperative findings suggested it was more likely to be a Rathke's pouch cyst than a true adenoma. Because the cyst was so large sampling was difficult and histology was unhelpful showing only crushed bone and damaged pituitary tissue.

She made reasonable post-operative recovery, needing to go back to theatre once due to excessive bleeding, but then recovered well after this. 4 months later the patient remained amenorrhoeic. Her implanon, which she had been using since 2005, was remove. She was found to have ongoing hyperprolactinaemia, suppressed gonadotrophs and a low oestradiol. The rest of her pituitary panel had normalised. The hyperprolactinaemia was put down to stalk damage as repeat MRI showed a reduction in size of remaining lesion without any ongoing signs of stalk compression. Cabergoline was trialled to treat the ongoing hypogonadotrophic hypogonadism, interestingly however, despite complete suppression of prolactin the patient remained completely amenorrhoeic. In 2012 The decision was made to commence HRT 
for bone protection. In February 2013 a total abdominal hysterectomy was performed so that HRT including oestrogen replacement could be safely commenced. The surgery and post-operative period went smoothly and HRT was commenced.

\section{Discussion}

Rathke's cleft cysts are often incidental lesions and are most frequently are asymptomatic. They only rarely present with neurological defects and endocrine dysfunction ${ }^{6}$.

A thorough search of available literature shows only one similar case published in 1978 in the JAMA. Trokoudes, et $\mathrm{al}^{1}$ report on a case of a young lady with subfertility and amenorrhoea thought to have a prolactinoma. She was successfully treated with bromocriptine and fell pregnant. She was operated on post partum and was actually found to have very large Rathke's pouch cyst and only a very small microadenomatous prolactin-secreting tumour for which to account for the effect of bromocriptine. Schittenhelm, et $\mathrm{al}^{5}$ describe and interesting case of intraoperative rupture of a Rathke's cyst causing post operative hypophysitis leading to visual change and amenorrhoea. In contrast to a direct pressure or compression causing dysfunction, this demonstrates pituitary tissue damage leading to similar symptoms. This could be a similar phenomenon to the pituitary tissue damage in the absence of compression accounting for ongoing hypogondatrophic hypogonadism in our case study.

A broader search for other pituitary and sellar lesions causing hypogona-dotrophic hypogonadism showed few similar published cases. Thomas, et $\mathrm{al}^{2}$ reported hypogondism as a part of a constellation of defects following radiation damage after radiation of a pituitary macroadenoma in a 23 year old lady. Kumar, et $\mathrm{al}^{3}$ report precocious puberty in a 15 year old female with recurrence of a supra-sellar arachnoid cyst which had previously been excised. Liao, et $\mathrm{al}^{4}$ reported an ACTH producing tumour in a 20 year old female mimicking PCOS by the androgen like effects of adrenal hormones.

\section{Conclusions}

Pituitary etiologies are an important cause of menstrual abnormalities and hypogonadism. Even non-functioning, or non-hormone producing, lesions can cause significant gondatrophin hormone imbalance purely from compression effect on the pituitary stalk. Definitive surgery may not fully correct hypogonadism caused by pituitary lesions.

\section{REFERENCES}

1. With hyperprolactinemia and a Rathke's pouch cyst. Journal of the American Medicak Association 1978; 240(5): 471-3.

2. Thomas VP, Sathya B, George S, Thomas N. Pregnancy in a patient with hypopituitarism following surgery and radiation for a pituitary adenoma. Journal of Postgraduate Medicine 2005; 51(3): 223-4.

3. Kumar R, Singhal N. Suprasellar arachnoid cyst with delayed puberty. Journal of the Indian Academy of Pediatrics 2007; 44(11): 858-60.

4. Liao CC, Lin SY, Lin HW, Chen KH, Chang LH, Chen ST, Wang J. Menstrual abnormalities in a woman with ACTH-dependent pituitary macroadenoma mimicking polycystic ovary syndrome. Taiwanese Journal of Obstetrics and Gynecology 2006; 45(2): 176-9.

5. Schittenhelm J, Beschorner R, Psaras T, Capper D, Nägele T, Meyermann R, Saeger W, Honegger J, Mittelbronn M. Rathke's cleft cyst rupture as potential initial event of a secondary perifocal lymphocytic hypophysitis: proposal of an unusual pathogenetic event and review of the literature. Neurosurgery Review 2008; 31(2): 157-63.

6. Zada G. Rathke cleft cysts: a review of clinical and surgical management. Journal of Neurosurgery 2011; 31(1): E1. 